\title{
THE
}

1981

\section{Magnetic Field Effects on the Spin Dynamics of the Linear Spin-1/2 Heisenberg Antiferromagnet}

\author{
Gerhard Müller \\ University of Rhode Island, gmuller@uri.edu \\ Harry Thomas \\ Hans Beck \\ Jill C. Bonner \\ University of Rhode Island
}

Follow this and additional works at: https://digitalcommons.uri.edu/phys_facpubs

Terms of Use

All rights reserved under copyright.

\section{Citation/Publisher Attribution}

Müller, G., Thomas, H., Beck, H., \& Bonner, J. C. (1981). Magnetic field effects on the spin dynamics of the linear spin-1/2 Heisenberg antiferromagnet. Solid State Commun., 38(1), 1-5. doi: 10.1016/ 0038-1098(81)91151-0

Available at: http://dx.doi.org/10.1016/0038-1098(81)91151-0

This Article is brought to you for free and open access by the Physics at DigitalCommons@URI. It has been accepted for inclusion in Physics Faculty Publications by an authorized administrator of DigitalCommons@URI. For more information, please contact digitalcommons-group@uri.edu. 


\title{
Magnetic Field Effects on the Spin Dynamics of the Linear Spin-1/2 Heisenberg Antiferromagnet
}

\author{
Gerhard Müller, ${ }^{1}$ Harry Thomas, 1 Hans Beck, ${ }^{2}$ and Jill C. Bonner 3 \\ 1 Institut für Physik, Universität Basel, $\mathrm{CH}-4056$ Basel, Switzerland \\ 2 Institut de Physique de l'Université, $\mathrm{CH}-2000$ Neuchâtel, Switzerland \\ 3 Department of Physics, University of Rhode Island, Kingston RI 02881, USA
}

\begin{abstract}
We present a new approach to the low temperature dynamics of a quantum Heisenberg antiferromagnetic chain, which employs a combination of techniques: exact finite chain calculations; exact Bethe Ansatz calculations; and exact sum rules and selection rules. A striking property of the selection rules is that the contribution of several classes of states to the dynamics in non-zero field for finite systems is shown to vanish in the thermodynamic limit. Many novel quantum field-dependent effects appear such as soft modes, and multiple peak structure in the structure factor, which should be experimentally observable.
\end{abstract}

Spin dynamical calculations on the one-dimensional (1D) Heisenberg antiferromagnet (HB AF)

$$
\mathcal{H}=J \sum_{l=1}^{N} \vec{S}_{l} \cdot \vec{S}_{l+1}-g \mu_{\mathrm{B}} H \sum_{l=1}^{N} S_{l}^{z}
$$

in zero and nonzero magnetic field have relied heavily on classical theory because of the relative ease of solvability of the classical as compared to the quantum HB AF. However evidence is increasing, both experimental and theoretical, that quantum effects are extremely important at low temperatures. The $S=\frac{1}{2} \mathrm{HB} \mathrm{AF}(1)$ has so far resisted rigorous analytic attack for both statics and dynamics. Semi-classical calculations or approximate many-body techniques have therefore been employed. In the belief that such approaches are ineffective in providing detailed information on the dynamics, we have pioneered a new approach $[1,2]$ which is based on four main techniques: (i) analytic calculations of excitation energies and densities of states of a special class of Bethe-Ansatz states; (ii) exact finite chain calculations of matrix elements for chains of 4 through 10 spins; (iii) derivation and application of two different kinds of selection rules; and (iv) the use of various kinds of sum rules. Major emphasis has been placed on zero temperature studies.

Results obtained for the $S=\frac{1}{2} \mathrm{HB}$ AF (1) in zero magnetic field have already been published $[1,2]$. Several novel features have been found which are purely quantum effects. The purpose of this short communication is to highlight many interesting results which appear when a magnetic field is applied. Spin dynamics of the 1D HB AF (1) in nonzero magnetic field is of current experimental interest as a result of very recent neutron scattering experiments on the quasi-lD AF compounds CPC $\left(S=\frac{1}{2}\right)[3]$ and TMMC $\left(S=\frac{5}{2}\right)[4]$ both in a field of $70 \mathrm{kOe}$. Active experimental investigation is also underway of proton spin-lattice relaxation rates in the quasi-ID $S=\frac{1}{2} \mathrm{AF}$ systems $\mathrm{CuSeO}_{4} \cdot 5 \mathrm{H}_{2} \mathrm{O}, \mathrm{CuSO}_{4} \cdot 5 \mathrm{H}_{2} \mathrm{O}[5]$ and $\alpha$-CuNSal [6]. This presentation is directed towards experimentalists in the hope of eventual experimental verification of the new features. A longer publication containing calculational details will appear in due course [7].

The quantity which contains moat detailed dynamical information and which is of direct experimental interest is the dynamic structure factor $S_{\mu \mu}(q, \omega)$, the Fourier transform of the dynamic two-spin correlation function $\left\langle S_{l}^{\mu}(t) S_{l+R}^{\mu}\right\rangle$. At $T=0$, the number of excitations contributing to $S_{\mu \mu}(q, \omega)$ is considerably reduced by selection rules which result from the symmetry properties of $\mathcal{H}$. The translational symmetry along the chain (due to periodic boundary conditions) allows for a classification of the eigenstates according to wave numbers $k=(2 \pi / N) n, n=0, \pm 1, \ldots$ and gives 
rise to the selection rule

$$
\left\langle k \ldots\left|S^{\mu}(q)\right| k^{\prime} \ldots\right\rangle \propto \delta_{k^{\prime}, k+q+2 \pi n}, \quad n=0, \pm 1, \ldots
$$

The rotational symmetry in spin-space allows for the introduction of the quantum number of the total spin $S^{T}$ and its z-component $S_{z}^{T}$, providing the following selection rules

$$
\begin{gathered}
\left\langle S^{T} \ldots\left|S^{\mu}(q)\right| S^{\prime T} \ldots\right\rangle=0 \quad \text { if } \quad\left|S^{T}-{S^{\prime}}^{T}\right|>1 \quad \text { or } \quad S^{T}={S^{\prime}}^{T}=0 \\
\left\langle S_{z}^{T} \ldots\left|S^{z}(q)\right| S^{\prime}{ }_{z}^{T} \ldots\right\rangle=0 \quad \text { unless } \quad S_{z}^{T}={S^{\prime}}_{z}^{T} \\
\left\langle S_{z}^{T} \ldots\left|S^{ \pm}(q)\right|{S^{\prime}}_{z}^{T} \ldots\right\rangle=0 \quad \text { unless } S_{z}^{T}={S^{\prime}}_{z}^{T} \pm 1 .
\end{gathered}
$$

These selection rules tell us which classes of states have nonzero matrix elements with the ground state (whose character changes, of course, with the magnetic field). For the special case of $h=0$ (we use a reduced field $\left.h=g \mu_{\mathrm{B}} H / J\right)$ the ground state is a singlet $\left(S^{T}=0\right)$. According to (3) the only contributing states to $S_{\mu \mu}(q, \omega)$ are triplets $\left(S^{T}=1, S_{z}^{T}=0, \pm 1\right)$. Let us consider the general case when at $h \neq 0$ the ground state has quantum numbers $S^{T}=S_{z}^{T}=N \sigma$, where $\sigma\left(0 \leq \sigma \leq \frac{1}{2}\right)$ is the magnetization. The selection rules (3)-(5) now allow six classes of excitations to contribute to $S_{\mu \mu}(q, \omega)$. At $h=0$ the six classes reduce to three classes. A most striking feature for $h \neq 0$ is the presence of additional selection rules with $N$-dependent coefficients which cause three out of the six classes to be excluded from the $h \neq 0$ dynamics in the thermodynamic limit. These selection rules for the "macroscopic system" are a consequence of the Wigner-Eckart theorem relating matrix elements involving states belonging to the same multiplet.

In the next step of our approach we are able to identify those excitations which predominate in $S_{\mu \mu}(q, \omega)$ as special eigenstates in Bethe's fformalism [8], to calculate their energies in the thermodynamic limit and to estimate their spectral weight distribution in $S_{\mu \mu}(q, \omega)$. Before coming to the more complex $h \neq 0$ case, let us briefly summarize the results for $h=0$. Here, the new approach yields an approximate analytic expression for $S_{\mu \mu}(q, \omega)$ which fits excellently into the fragmentary picture of the few available exact results $[1,2]$ :

$$
S_{\mu \mu}(q, \omega)=A \frac{\Theta(\omega-(\pi J / 2) \sin q) \Theta(\pi J \sin (q / 2)-\omega)}{\sqrt{\omega^{2}-(\pi J / 2)^{2} \sin ^{2} q}},
$$

where $\Theta$ is the step function and $A$ is a constant of order unity. $S_{\mu \mu}(q, \omega)$ is governed by a twoparameter continuum of spin-wave-type triplet $\left(S^{T}=1\right)$ excitations. We call them spin-wave continua SWC 1, 3, 4, depending on the component of $S_{\mu \mu}$ involved. Expression (6) "switches on" at the lower continuum boundary $\epsilon_{1}(q)=(\pi J / 2) \sin q$ (first derived by Des Cloizeaux and Pearson [9]), where there is a divergence. A tail of decreasing spectral weight extends to the upper continuum boundary $\left.\epsilon_{2}(q)=\pi J \sin (q / 2)\right)$, where there is a cut-off. This expression is not rigorous, but exact sum rules are violated by only a small amount. A plot of $S_{z z}(q, \omega)$ for two fixed values of $q$ is shown as Fig. 2a of Ref. [1]. Expression (6) predicts increasing asymmetry in the spectral weight distribution as $q \rightarrow \pi$, which has recently been verified by neutron scattering data (see Fig. 7 of Ref. [3]). This asymmetry is a quantum effect, since a classical spin-wave calculation would result in a delta function at the classical spin-wave frequency. The integrated intensity derived from (6) is in much better agreement with neutron scattering data on CPC [10] than the semiclassical result.

For nonzero magnetic field the longitudinal structure factor $S_{z z}(q, \omega)$ behaves differently from the transverse counterparts, $S_{x x}(q, \omega) \equiv S_{y y}(q, \omega)$. In comparison with the classical model where the system enters a spin-flop state for $h>0$, the $S=\frac{1}{2}$ system displays behavioral anomalies in the dynamics in the limit $h \rightarrow 0^{+}$. Even more drastic differences occur between the classical spinwave dispersion spectra and the lowest excitation branches of the $S=\frac{1}{2}$ chain as $h$ progressively increases from zero to $h=h_{\mathrm{c}}=2$. For example, the classical transverse spin-waves develop a gap at $q=0$ and the $h=0$ sine curve distorts into a cosine-like curve at $h=h_{\mathrm{c}}$ [3]. In the quantum 
case, on the other hand, we have obtained for the lowest-lying excitations contributing to $S_{x x}(q, \omega)$ the dispersion curve

$$
\omega(q)=2 D(h)\left|\cos \frac{q}{2} \sin \left(\frac{q}{2}-\pi \sigma(h)\right)\right|
$$

with $2 D(h)=\pi+h(1-\pi)$, in qualitative agreement with Pytte's result in a Hartree-Fock approximation [11] and with Ishimura and Shiba's numerical result based on an exact calculation [12]. A striking non-classical feature of (7) is the existence of a zero-frequency mode which progressively moves from the zone center $(q=0)$ to the zone boundary $(q=\pi))$ as the field increases from $h=0$ to $h=h_{\mathrm{c}}$. Moreover, our calculations make clear that (7) is only the lower boundary of a two-parameter continuum of excitations. A careful analysis shows that there are in total six continua of excitations, SWC 1 to SWC 6, contributing to $S_{\mu \mu}(q, \omega)$ for $h \neq 0$ and $N$ finite [7]. As a consequence of the selection rules for the "macroscopic system" only three continua (SWC 2, 3, 6) survive in the thermodynamic limit [7].

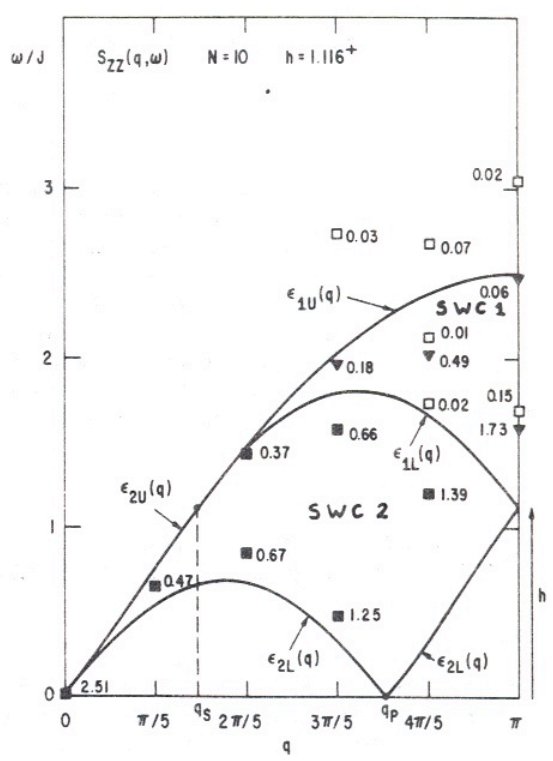

Figure 1. Spectral representation of $S_{z z}(q, \omega)$ for the HBAF with $N=10$ and $h \gtrsim 1.116$. For each value of the wave number $q=2 \pi r / 10, r=0,1, \ldots, 5 ; S_{z z}(q, \omega)$ is a sum of delta functions. Full symbols denote "Bethe Ansatz" states and open symbols "anomalous" states. The numbers give the corresponding spectral weights. Excitations not shown have spectral weights smaller by at least an order of magnitude. The full lines represent the boundaries of SWC 1 and SWC 2 .

Let us consider the longitudinal fluctuations in more detail. Fig. 1 shows the excitations for $N=10$ which contribute to $S_{z z}(q, \omega)$ in a field somewhat greater than half the critical field. By solid triangles and solid squares we have distinguished those excitations which exhaust most of the spectral weight, and for which we can calculate the energies in the framework of Bethe's formalism. In the thermodynamic limit they form the continua SWC 1 and SWC 2 with lower and upper boundaries shown as solid lines in Fig. 1. In Fig. 2 we have sketched how the shapes of SWC 1 and SWC 2 change as the field varies over the complete range from $h=0$ to $h=h_{\mathrm{c}}=2$. In zero field SWC 2 vanishes and SWC 1 dominates $S_{z z}(q, \omega)$ for both finite and infinite $N$. At $h \neq 0$ SWC 2 appears with zero-frequency modes at $q=0$ and $q=\pi(1-2 \sigma)$. Note that as soon as the smallest field is applied, the broad SWC 1 is completely suppressed in the infinite system, leaving only the fairly narrow SWC 2. The effects of this dramatic limiting process as $h \rightarrow 0^{+}$are, however, masked to some extent by "anomalous" states appearing in $S_{z z}(q, \omega)$ which lie beyond the upper boundary of SWC 2 [7]. For $N=10$ they are denoted as open squares in Fig. 1. Experimentally observable features are the "soft mode" at $q_{p}=\pi(1-2 \sigma)$ and the gap at $q=\pi$ with energy $E_{\text {gap }} / J=h$. 

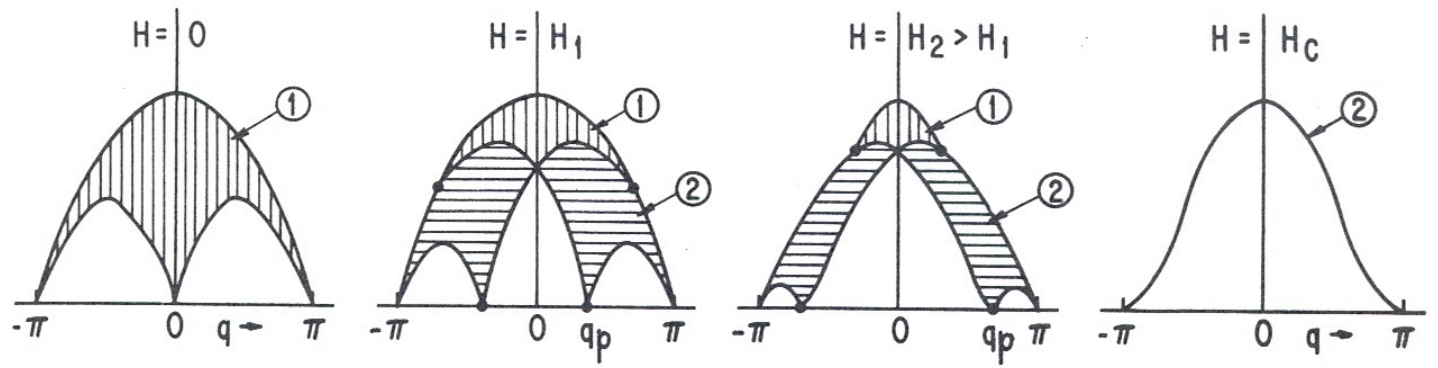

Figure 2. The two SWC contributing to $S_{z z}(q, \omega)$ shown schematically as $H$ varies between 0 and $H_{\mathrm{c}}$.

Let us now briefly discuss the transverse fluctuations. Here we have found that there are four SWC's contributing to $S_{x x}(q, \omega)$ for finite $N$, and only two of these survive in the thermodynamic limit. Details are given in Ref. [7]. The surviving continuum SWC 3 has zero frequency modes at $q=q_{\mathrm{s}}=2 \pi \sigma$ and $q=\pi$ as also does SWC 6 (SWC4 and 5 vanish [7]). At $q=0$ the spectrum has a gap of the same magnitude as the gap appearing in $S_{z z}(q, \omega)$ at $q=\pi$. These characteristic features should be experimentally observable.

An important quantity for experimental observation is the integrated intensity. Figs. 3 and 4 show $I_{z z}(q)$ and $I_{x x}(q)$, respectively, at $T=0$ for various values of the magnetic field, as obtained from finite-chain calculations. Fig. 3 shows the relative importance of the various modes contributing to the longitudinal fluctuations. At $h=0$, the $q=\pi$ mode is dominant, giving rise to a logarithmic divergence in $I_{z z}(q)$. With increasing field, the dominant mode corresponds to $q=\pi(1-2 \sigma)$. The singularity for $h \neq 0$ appears to be weaker (finite cusp) than for $h=0$. The dashed curve is our prediction for $\mathrm{CPC}\left(H_{\mathrm{c}} \simeq 330 \mathrm{kOe}\right)$ in a field of $70 \mathrm{kOe}$. Fig. 4 shows $I_{x x}(q)$,

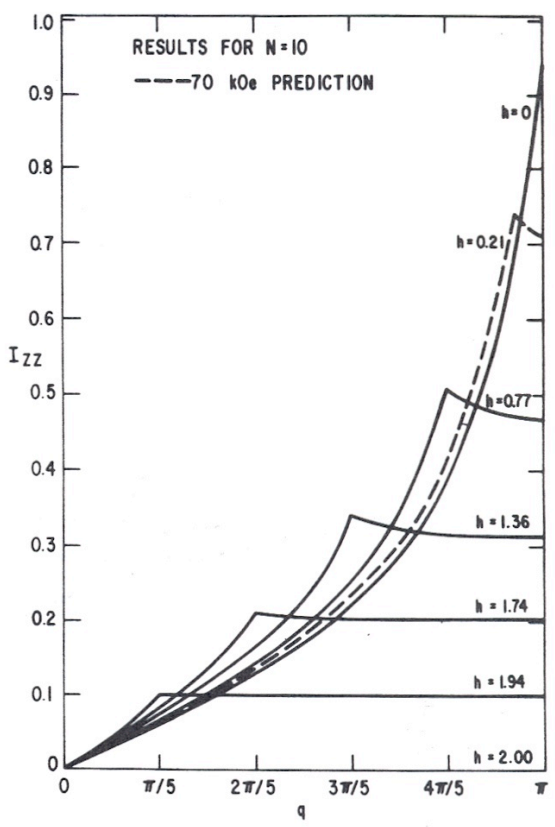

Figure 3. Finite chain results $(N=10)$ for the $T=0$ longitudinal integrated intensity at various reduced fields (solid lines). The dashed line is an interpolation for the special field $h=0.21$ corresponding to $70 \mathrm{kOe}$ in $\mathrm{CPC}$. 


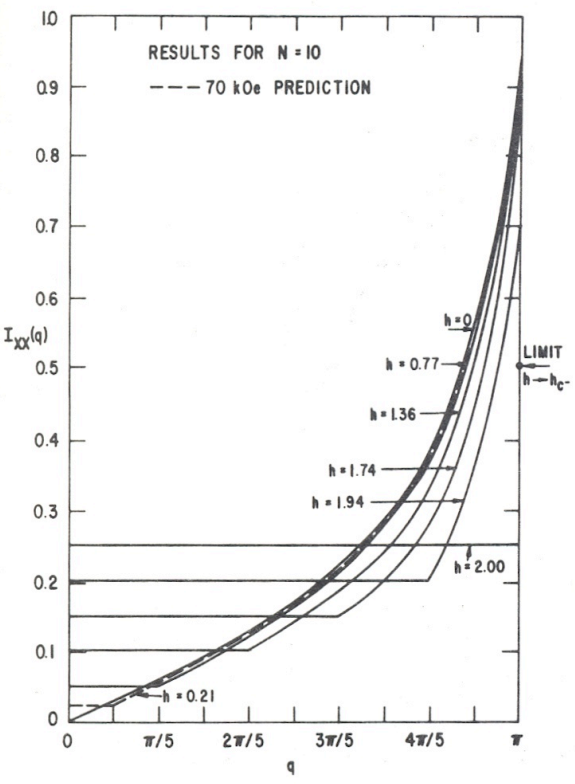

Figure 4. Finite chain results $(N=10)$ for the $T=0$ transverse integrated intensity at various reduced fields (solid lines). The dashed line is an interpolation for the special field $h=0.21$.

the integrated intensity of the fluctuations perpendicular to the field. Whereas the dominant mode in $\left.I_{z z}(q)\right)$ moves away from the zone boundary with increasing field, the dominant mode in $I_{x x}(q)$ remains at $q=\pi$ independent of $h$, although its weight decreases as $h$ becomes larger. Again the dashed curve shows our prediction for $\mathrm{CPC}$ at $\mathrm{H}=70 \mathrm{kOe}$.

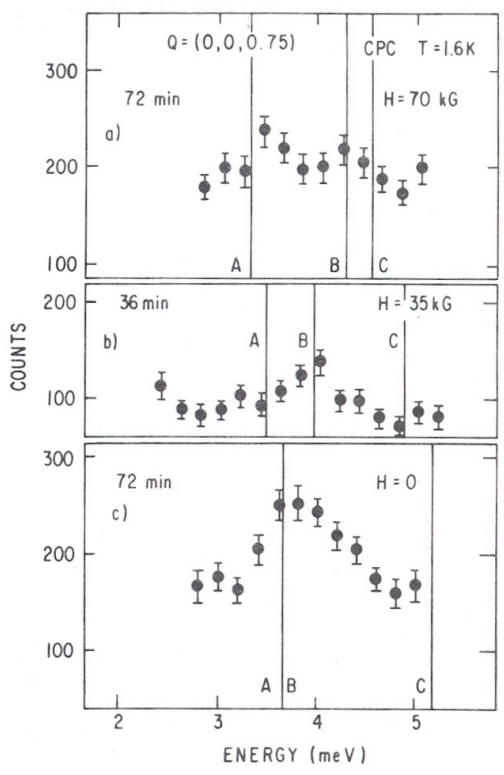

Figure 5. Comparison of theoretical predictions for the peak positions in $S_{x x}(q, \omega)$ with corresponding experimental data for CPC by Heilmann et al. (see Ref. [3]). The three scans at $q=\pi / 2$ are for reduced fields $h=0.21, h=0.105$ and $h=0$, respectively. The vertical lines represent special energies corresponding to continuum boundaries. 
Fig. 5 compares our theory with low $T$ inelastic neutron scattering measurement on CPC at zero field, and in fields of $35 \mathrm{kOe}$ and $70 \mathrm{kOe}$ [3]. Crystal orientation appears to correspond to our $S_{x x}(q, \omega)$. The vertical lines denoted A, C correspond to energies of the lower and upper boundaries of SWC 3 and SWC 6, where our theory predicts discontinuities in the $T=0 S_{x x}(q, \omega)$. Line B represents approximately the upper boundary of the non-negligible "anomalous" states associated with SWC 6 [7].

Fig. 5a corresponds to CPC data at $70 \mathrm{kOe}$. They reveal two peaks which both are well understood by our theory. The peak at the lower energy corresponds to the lower boundary of SWC 3, 6 given by line $\mathrm{A}$ at $3.2 \mathrm{meV}$. The peak at higher energy is clearly due to the combined effect of the special energies and the upper boundary of SWC 3, shown as lines Band C at $4.2 \mathrm{meV}$ and 4.5 meV, respectively.

In Fig. 5b comparison is made with the data at $35 \mathrm{kOe}$, yielding also fair agreement with our theory. At this field the lines $\mathrm{B}$ and $\mathrm{C}$ are more separated and the data indicate in addition to the peaks corresponding to lines $\mathrm{A}(3.45 \mathrm{meV})$ and $\mathrm{B}(3.9 \mathrm{meV})$ a third additional weak signal, corresponding to the line $\mathrm{C}$ at $4.8 \mathrm{meV}$.

At $h=0$ the maximum intensity is expected to be close to the des Cloizeaux-Pearson energy of $3.63 \mathrm{meV}$ as required by expression (6) and as verified in Fig. 5c (lines A,B).

Finally we should comment on recent work by Ishimura and Shiba [13], which covers some of the same ground here discussed. However Ishimura and Shiba do not take account of upper continuum boundaries, and fail to be aware of the fact that certain classes of states lose their weight in the thermodynamic limit.

\section{Acknowledgments}

We are happy to acknowledge stimulating and clarifying discussion with I. Heilmann, G. Shirane, M. Puga, J. Axe and P. Hohenberg. JCB expresses appreciation to the Institutes at Basel and Neuchâtel for their hospitality. Work supported by the Swiss National Science Foundation and, in part for JCB, by the U.S. National Science Foundation, grant No. DMR-24136. We have used a modified cmpj.sty style file.

\section{References}

1. G. Müller, H. Beck and J.C. Bonner, Phys. Rev. Lett. 43, 75 (1979).

2. G. Müller, H. Thomas and H. Beck, in Ordering in Strongly Fluctuating Condensed Matter Systems, Ed. T. Riste, p. 151, Plenum Publ. Corp. (1980).

3. I.U. Heilmann, G. Shirane, Y. Endoh, R.J. Birgeneau, and S.L. Holt, Phys. Rev. B 18, 3530 (1978).

4. l.U. Heilmann R.J. Birgeneau, Y. Endoh, G. Reiter, G. Shirane and S.L. Holt, Solid State Comm. 31, 607 (1979).

5. J.P. Groen, T.O. Klaassen, N.J. Poulis, G. Müller, H. Thomas and H. Beck, Phys. Rev. B 22, 5369 (1980).

6. L.J. Azevedo, A. Narath, P.M. Richards and Z.G. Soos, Phys. Rev. B 21, 2871 (1980).

7. G. Müller, H. Thomas, H. Beck and J.C. Bonner, submitted to Phys. Rev. B.

8. H.A. Bethe, Z. Physik 71, 205 (1931).

9. J. des Cloizeaux and J.J. Pearson, Phys. Rev. 128, 2131 (1962).

10. Y. Endoh, G. Shirane, R.J. Birgeneau, P.M. Richards, and S.L. Holt, Phys. Rev. Lett. 32, 170 (1974).

11. E. Pytte, Phys. Rev. B 10, 4637 (1974).

12. N. Ishimura and H. Shiba, Progr. Theor. Phys. 57, 1862 (1977).

13. N. Ishimura and H. Shiba, preprint. 